\title{
Differential cross sections for target excitation and positronium formation in positron-helium scattering
}

\author{
Puspitapallab Chaudhuri and Sadhan K. Adhikari \\ Instituto de Física Teórica, Universidade Estadual Paulista, 01.405-900 São Paulo, São Paulo, Brazil
}

(Received 9 July 1997)

\begin{abstract}
Positronium formation and target excitation in positron-helium scattering have been investigated using the close-coupling approximation with realistic wave functions for the positronium and helium atoms. The following eight states have been used in the close-coupling scheme: $\operatorname{He}(1 s 1 s), \operatorname{He}\left(1 s 2^{1} s\right), \operatorname{He}\left(1 s 2^{1} p\right), \operatorname{He}\left(1 s 3^{1} s\right)$, $\mathrm{He}\left(1 s 3^{1} p\right), \operatorname{Ps}(1 s), \operatorname{Ps}(2 s)$, and $\operatorname{Ps}(2 p)$, where Ps stands for the positronium atom. Calculations are reported of differential cross sections for elastic scatering, inelastic target excitation to $\operatorname{He}\left(1 s 2{ }^{1} s\right)$ and $\operatorname{He}\left(1 s 2^{1} p\right)$ states, and rearrangement transition to $\operatorname{Ps}(1 s), \operatorname{Ps}(2 s)$, and $\operatorname{Ps}(2 p)$ states for incident positron energies between 40 and $200 \mathrm{eV}$. The coincidence parameters for the transition to the $\mathrm{He}\left(1 s 2^{1} p\right)$ state of helium are also reported and briefly discussed. [S1050-2947(98)05101-4]

PACS number(s): $34.90 .+\mathrm{q}$
\end{abstract}

\section{INTRODUCTION}

Of late, there is a renewed interest in positron-atom scattering due to the improvement in the nature of positron sources and the positron beam [1]. Among all positron-atom systems, the positron-helium system is of special interest to experimentalists $[2,3]$ and is widely studied as it has all the complicated features of a many-body system, but few-body techniques can be employed conveniently as in the positronhydrogen system. Moreover, due to the difficulty in obtaining an atomic hydrogen target, the experimental activity is limited in the case of positron-hydrogen scattering. On the other hand, there have been many experimental studies of target excitation and positronium formation in low and medium energy positron-helium scattering.

Besides the experimental works $[2,3]$, there are numerous theoretical studies of positron-helium scattering using various theoretical methods, such as the classical trajectory Monte carlo technique (CTMC) [4], the random phase approximation [5], the distorted wave approximation [6], and the close-coupling approximation (CCA) [7-13]. This allows us to compare different theoretical treatments with experiment and assess their usefulness. Of all the approximation schemes mentioned above, only the CCA fully includes the effect of coupling between various target states. The CCA provides a practical framework for treating electron-atom or positron-atom scattering and handles the elastic, excitation, and rearrangement channels in a unified way. It is of use in positron-atom scattering for both small and large atoms [14] and yields a calculational scheme which can, in principle, be improved upon by including more functions in the basis set.

We perform a detailed study of medium energy positronhelium scattering in the framework of eight-state CCA including two dynamically active electrons and using realistic wave functions for the helium and positronium (Ps) atom states for incident positron energy between $40 \mathrm{eV}$ and 200 $\mathrm{eV}$. The eight states included in the present CCA study are as follows: $\operatorname{He}(1 s 1 s), \operatorname{He}\left(1 s 2^{1} s\right), \operatorname{He}\left(1 s 2^{1} p\right), \operatorname{He}\left(1 s 3^{1} s\right)$, $\operatorname{He}\left(1 s 3^{1} p\right), \operatorname{Ps}(1 s), \operatorname{Ps}(2 s)$, and $\operatorname{Ps}(2 p)$. Most of the other theoretical studies on this system only presented results for angle-integrated partial cross sections for elastic and different target inelastic channels. Here, we present results for different differential cross sections and coherence parameters not only for the relevant elastic and inelastic channels but also for the rearrangement channels.

Recently, we have reported results for angle-integrated partial cross sections for elastic, inelastic, and Ps-formation cross sections at medium energies for positron-helium scattering $[11,12]$ using the above-mentioned CCA scheme for incident positron energies between $25 \mathrm{eV}$ and $400 \mathrm{eV}$. The calculated cross sections were in good agreement with the available experimental results. Two prominent $S$-wave resonances have also been reported in the positron-helium system at $19.27 \mathrm{eV}$ (width $0.001 \mathrm{eV}$ ) [12] and at $30 \mathrm{eV}$ (width $2-3 \mathrm{eV}$ ) [13]. However, a study of the differential cross sections and the coincidence parameters is necessary for a complete description of scattering. Here we report the differential cross sections for elastic scattering, inelastic target excitation to $\mathrm{He}\left(1 s 2^{1} s\right)$ and $\mathrm{He}\left(1 s 2^{1} p\right)$ states, and rearrangement transition to $\operatorname{Ps}(1 s), \operatorname{Ps}(2 s)$, and $\operatorname{Ps}(2 p)$ states for incident positron energies between 40 and $200 \mathrm{eV}$. We also present the results for the coincidence parameters, $\lambda$ and $\chi$ for excitation to the $\mathrm{He}\left(1 s 2^{1} p\right)$ state.

The positron-helium system is one of the simplest systems where the total capture cross sections to the Ps atom states are comparable to elastic and inelastic cross sections. Hence an ab initio dynamical description of the Ps formation in this system using realistic wave functions is most welcome. However, because of the two-centered nature of the final state in the Ps-formation channel and of the presence of two active electrons, the theoretical description of Ps-formation in positron-helium scattering is a difficult task. This is why most of the CCA calculations for this system have either neglected the Ps-formation states $[9,10]$ or treated them approximately by employing an effective one-electron model for helium and also simplified wave functions for the positronium atom [8].

In Sec. II we present a theoretical description of our study. We present the details of the wave functions as well as the partial-wave scattering Lippmann-Schwinger-type CCA 
equations used in the present study. In Sec. III we report the numerical results for differential cross sections for elastic, inelastic, and rearrangement collisions and for coincidence parameters for inelastic transition to the $\mathrm{He}\left(1 s 2^{1} p\right)$ state. Finally, in Sec. IV we present a summary of our study.

\section{THEORY}

In the CCA, the total wave function for the positronhelium system is written as

$$
\begin{aligned}
\Psi\left(\vec{r}_{1}, \vec{r}_{2}, \vec{r}_{3}\right)= & \sum_{\nu} \Phi_{\nu}\left(\vec{r}_{1}, \vec{r}_{2}\right) F_{\nu}\left(\vec{r}_{3}\right) \chi_{1}(3,21) \\
& +\sum_{\kappa} \Phi_{1 s}^{+}\left(\vec{r}_{1}\right) \eta_{\kappa}\left(\vec{r}_{23}\right)\left[G_{\kappa}^{P}\left(\vec{R}_{23}\right) \chi_{1}(1,23)\right. \\
& \left.+G_{\kappa}^{O}\left(\vec{R}_{23}\right) \chi_{2}(1,23)\right]+\sum_{\kappa} \Phi_{1 s}^{+}\left(\vec{r}_{2}\right) \eta_{\kappa}\left(\vec{r}_{13}\right) \\
& \times\left[G_{\kappa}^{P}\left(\vec{R}_{13}\right) \chi_{1}(2,13)-G_{\kappa}^{O}\left(\vec{R}_{13}\right) \chi_{2}(2,13)\right],
\end{aligned}
$$

with $r_{i j}=\left|\vec{r}_{i}-\vec{r}_{j}\right|, \quad \vec{R}_{i j}=\left|\vec{r}_{i}+\vec{r}_{j}\right| / 2$, where $\vec{r}_{i}(i=1,2)$ are the coordinates of the atomic electrons and $\vec{r}_{3}$ is the positron coordinate. Here $\Phi_{\nu}\left(\vec{r}_{1}, \vec{r}_{2}\right)$ and $\eta_{\kappa}\left(\vec{r}_{i j}\right)$ are the $\nu$ th and $\kappa$ th eigenstates of the helium and the Ps atoms, respectively. The ground-state wave function of the residual helium ion after positronium formation is $\Phi_{1 s}^{+}\left(\vec{r}_{i}\right)(i=1,2) ; F_{\nu}\left(\vec{r}_{3}\right)$ describes the motion of the incident positron and $G_{\kappa}\left(\vec{R}_{i j}\right)$ describes the relative motion between the Ps atom and the helium ion. The superscripts $O$ and $P$ on $G$ denote the Ps atom in the spintriplet (ortho) and spin-singlet (para) states, respectively; $\chi_{1}$ and $\chi_{2}$ are appropriate spin functions. The capture probability of a positron by an atomic electron in the ortho state is three times that in the para state, so that $G^{O}=\sqrt{3} G^{P}$, and these two possibilities are summed in our treatment.

The wave function $\Psi\left(\vec{r}_{1}, \vec{r}_{2}, \vec{r}_{3}\right)$ satisfies the following Schrödinger equation:

$$
(H-E) \Psi=0
$$

where the full Hamiltonian $H$ of the system is given by

$$
H=\sum_{i=1}^{2}\left(-\frac{1}{2 \mu} \nabla_{\vec{r}_{i}}^{2}-\frac{Z e^{2}}{r_{i}}\right)+\frac{e^{2}}{r_{12}}+H_{T},
$$

with

$$
H_{T}=\left(-\frac{1}{2 \mu} \nabla_{\vec{r}_{3}}^{2}+\frac{Z e^{2}}{r_{3}}\right)-\sum_{i=1}^{2} \frac{e^{2}}{r_{3 i}} .
$$

After a partial-wave projection, the coupled LippmannSchwinger-type scattering integral equations of the CCA become [7]

$$
\begin{aligned}
T_{\beta \alpha}^{J}\left(\tau^{\prime} k^{\prime}, \tau k\right)= & B_{\beta \alpha}^{J}\left(\tau^{\prime} k^{\prime}, \tau k\right)+\frac{1}{2 \pi^{2}} \sum_{\kappa} \int k^{\prime \prime} d k^{\prime \prime} \\
& \times \frac{B_{\beta \kappa}^{J}\left(\tau^{\prime} k^{\prime}, \tau^{\prime \prime} k^{\prime \prime}\right) T_{\kappa \alpha}^{J}\left(\tau^{\prime \prime} k^{\prime \prime}, \tau k\right)}{k^{\prime \prime 2}-k_{\kappa}^{2}-i 0},
\end{aligned}
$$

with

$$
\begin{aligned}
B_{\beta \alpha}^{J}\left(\tau^{\prime} k^{\prime}, \tau k\right)= & \frac{\left(k k^{\prime}\right)^{1 / 2}}{2 J+1} \sum_{\mathcal{M M M ^ { \prime } m m ^ { \prime }}}\left\langle L^{\prime} l^{\prime} M^{\prime} m^{\prime} \mid J \mathcal{M}\right\rangle \\
& \times\langle L l M m \mid J \mathcal{M}\rangle \int d \vec{k} d \vec{k}^{\prime} Y_{L^{\prime} M^{\prime}}^{*}\left(\hat{k}^{\prime}\right) \\
& \times Y_{L M}(\hat{k}) B_{\beta \alpha}\left(\vec{k}^{\prime}, \vec{k}\right) .
\end{aligned}
$$

Here $T_{\beta \alpha}^{J}\left(\tau^{\prime} k^{\prime}, \tau k\right)$ is the $t$ matrix for transition from channel $\alpha$ to $\beta, k$ and $k^{\prime}$ are the relevant wave numbers, $L$ and $M$ are the orbital angular momentum and the projection quantum number of the relative motion, respectively, $l$ and $m$ are the angular momentum and the projection quantum number of the atomic states, respectively, and $\tau$ stands for the collective quantum numbers $(n, l, m)$ of the atomic states, $n$ being the principal quantum number. The off-shell parts of the CCA Born terms for all the rearrangement channels are included in the calculation.

The elastic unitarity relation is given by

$$
\operatorname{Im}\left[T_{11}^{J}(k, k)\right]=\frac{1}{4 \pi}\left|T_{11}^{J}(k, k)\right|^{2},
$$

where Im stands for the imaginary part and the elastic channel is denoted by suffix $\alpha=\beta=1$.

The helium wave functions used in the present calculation are taken from Ref. [15]. These wave functions are fairly realistic and produce accurate energies not only for the ground state but also for the excited states of the helium atom. Of the different ground-state wave functions presented there, we used the one given by Eq. (17). Exact analytic wave functions were used for the Ps atom states.

The partial-wave CCA equations (2.5) for the $t$ matrix were solved by the standard matrix-inversion technique $[7,16]$. This yields the various on-shell scattering amplitudes in the momentum space. The differential cross sections have been obtained by using the standard relations.

In addition to calculating the differential cross sections, we also calculated the positron impact coherence parameters for the excitation to the $\mathrm{He}\left(1 s 2^{1} p\right)$ state of the helium atom and rearrangement transition to the $\operatorname{Ps}(2 p)$ state of the positronium atom. There have been previous calculations of coherence parameters for excitation to the $\operatorname{He}\left(1 s 2^{1} p\right)$ state of the helium atom in electron-helium and positron-helium scattering, respectively $[10,17]$.

For a detailed understanding of the transition to a non$s$-wave atomic state, for example, the $\mathrm{He}\left(1 s 2^{1} p\right)$ state of helium, in addition to the differential cross section, a study of the coherence parameters is necessary. In this case the final state is a coherent superposition of degenerate magnetic sublevels:

$$
|\Psi\rangle=a_{1}|11\rangle+a_{0}|10\rangle+a_{-1}|1-1\rangle
$$




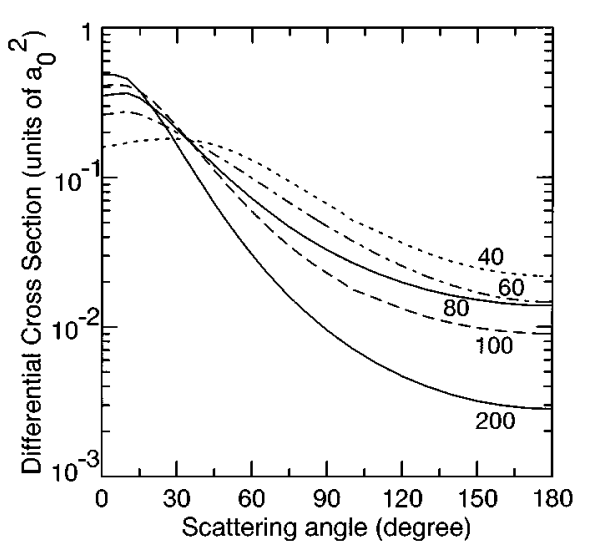

FIG. 1. Differential cross sections (in units of $a_{0}^{2}$ ) for elastic scattering of helium by positron at different incident energies in the CCA approach. The curves are labeled by different incident energies.

where the amplitude $a_{\mathcal{M}}$ describes the excitation to the particular sublevel $|J=1, \mathcal{M}\rangle$ of the $\mathrm{He}\left(1 s 2^{1} p\right)$ state. Assuming azimuthal symmetry of the scattering process, one has $a_{1}=-a_{-1}$. The differential cross section for the excitation of the $\operatorname{He}\left(1 s 2^{1} p\right)$ state is the sum of the three possibilities and is given by

$$
\sigma=\sum_{\mathcal{M}=-1}^{1}\left|a_{\mathcal{M}}\right|^{2}=2\left|a_{1}\right|^{2}+\left|a_{0}\right|^{2},
$$

where $\left|a_{\mathcal{M}}\right|^{2}$ is the differential cross section for exciting the angular momentum state of projection $\mathcal{M}$.

The amplitudes $a_{\mathcal{M}}, \mathcal{M}=-1,0,1$ are the outputs of the dynamical CCA calculation. In general, these amplitudes are complex. The differential cross section (2.9) does not provide completely detailed information about these amplitudes. Two more parameters $\lambda$ and $\chi$ have been introduced for this purpose. These dimensionless parameters, in addition to the differential cross section, provide a better description of the final state of scattering. The parameter $\chi$ provides the relative phase between $a_{1}$ and $a_{0}$ and is defined by [17]

$$
\frac{a_{1}}{a_{0}}=\left|\frac{a_{1}}{a_{0}}\right| \exp (i \chi) .
$$

The parameter $\lambda$ is defined by

$$
\lambda=\frac{\left|a_{0}\right|^{2}}{2\left|a_{1}\right|^{2}+\left|a_{0}\right|^{2}} .
$$

In the present study we calculate these parameters for inelastic excitation to the $\mathrm{He}\left(1 s 2^{1} p\right)$ state.

\section{NUMERICAL RESULTS}

\section{A. Differential cross section}

The momentum-space CCA equations were first discretized by using Gauss quadrature points and then solved by the matrix-inversion technique. Some 50 Gauss points were needed in each channel for obtaining convergence. In $S$ wave, the eight-state CCA equations lead to a complex ma-
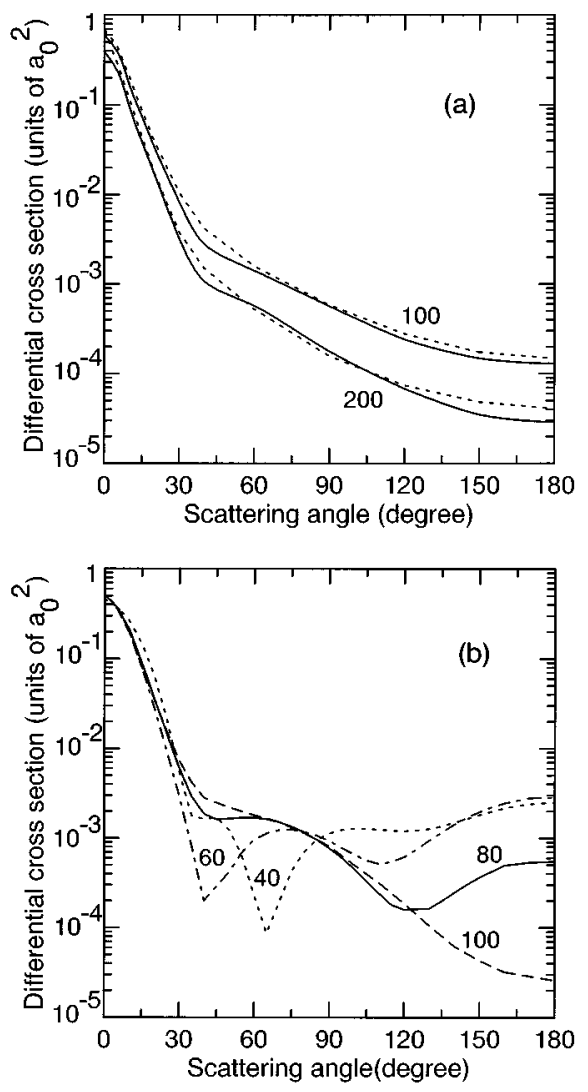

FIG. 2. Differential cross sections (in units of $a_{0}^{2}$ ) for excitation to the $\mathrm{He}\left(1 s 2^{1} s\right)$ state in positron-helium scattering employing the following CCA basis sets: (a) $\operatorname{He}(1 s 1 s), \operatorname{He}\left(1 s 2^{1} s\right)$, and $\mathrm{He}\left(1 s 2^{1} p\right)$ states; (b) $\mathrm{He}(1 s 1 s), \quad \mathrm{He}\left(1 s 2^{1} s\right), \quad \mathrm{He}\left(1 s 2^{1} p\right)$, $\mathrm{He}\left(1 s 3^{1} s\right), \operatorname{He}\left(1 s 3^{1} p\right), \operatorname{Ps}(1 s), \operatorname{Ps}(2 s)$, and $\operatorname{Ps}(2 p)$ states. The curves are labeled by the energy of the incident positron. In (a) the solid lines are the present results and the dotted lines are taken from Willis et al. [10].

trix equation of dimension 400. In non- $S$ waves, the dimension of the complex matrix equation is 550 .

The results for angle-integrated partial elastic, inelastic, and rearrangement cross sections of positron-helium scattering were reported in Ref. [12]. For obtaining convergence of the angle-integrated cross section, 12 (15) angular momenta $J$ states were needed for energies up to $80 \mathrm{eV}(200 \mathrm{eV})$. For the differential cross sections and $\lambda$ and $\chi$ parameters it was more difficult to obtain convergence. For elastic scattering and rearrangement scattering to the $\operatorname{Ps}(1 s)$ state, 15 angular momenta were needed for obtaining convergence. For transitions to $\mathrm{He}\left(1 s 2^{1} s\right), \mathrm{He}\left(1 s 2^{1} p\right), \mathrm{He}\left(1 s 3^{1} s\right), \mathrm{He}\left(1 s 3^{1} p\right)$ states of helium and $\operatorname{Ps}(2 s)$ and $\operatorname{Ps}(2 p)$ states of positronium, 30 angular momentum states were employed. The contributions of higher partial waves not included in the CCA dynamical calculations were approximated by the respective Born terms and added to the results of the dynamical calculations.

First, we present the results for various differential cross sections. In particular, we consider elastic and inelastic cross sections to $\mathrm{He}(1 s 1 s), \mathrm{He}\left(1 s 2^{1} s\right), \mathrm{He}\left(1 s 2^{1} p\right), \mathrm{He}\left(1 s 3^{1} s\right)$, and $\operatorname{He}\left(1 s 3^{1} p\right)$ states of helium and rearrangement cross sections to $\operatorname{Ps}(1 s), \operatorname{Ps}(2 s)$, and $\operatorname{Ps}(2 p)$ states of the positronium atom. Differential cross sections for transitions to $\mathrm{He}\left(1 s 3^{1} s\right)$ and $\mathrm{He}\left(1 s 3^{1} p\right)$ states are found to be negligible 


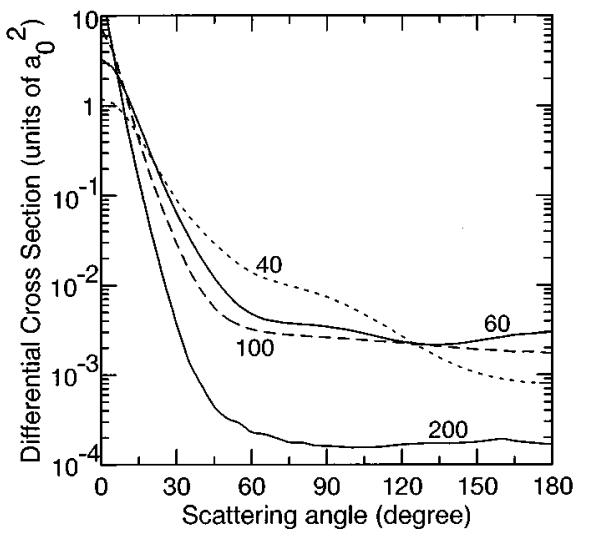

FIG. 3. Differential cross sections (in units of $a_{0}^{2}$ ) for excitation to the $\mathrm{He}\left(1 s 2^{1} p\right)$ state in positron-helium scattering employing the present eight-state CCA scheme. The curves are labeled by different incident positron energies.

in comparison to that for $\operatorname{He}\left(1 s 2^{1} s\right)$ and $\operatorname{He}\left(1 s 2^{1} p\right)$ states. Hence, these transitions are not considered here.

In order to show the general trend of the result, we performed calculations at the following incident positron energies: $40 \mathrm{eV}, 60 \mathrm{eV}, 80 \mathrm{eV}, 100 \mathrm{eV}$, and $200 \mathrm{eV}$. The differential cross sections for elastic scattering of helium by positron obtained by the eight-state CCA scheme are plotted in Fig. 1.

Next, we present results for different inelastic differential cross sections. There have been previous calculations for inelastic transitions to the $\mathrm{He}\left(1 s 2^{1} s\right)$ state employing the three-state CCA scheme with the states $\mathrm{He}(1 s 1 s)$, $\mathrm{He}\left(1 s 2^{1} s\right)$, and $\mathrm{He}\left(1 s 2^{1} p\right)$ [10]. Hence, we find it appropriate to compare our results with those of Ref. [10] employing the same three states of helium and we do that in Fig. 2(a). The agreement between the two calculations at 100 and $200 \mathrm{eV}$ is very satisfactory. In Fig. 2(b) we present the results of the present eight-state CCA calculation for inelastic transitions to the $\mathrm{He}\left(1 s 2^{1} s\right)$ state. In Fig. 3 we plot the differential cross section for excitation to the $\mathrm{He}\left(1 s 2^{1} p\right)$ state in positron-helium scattering employing the present eightstate CCA. We find that, in general, the three-state results are

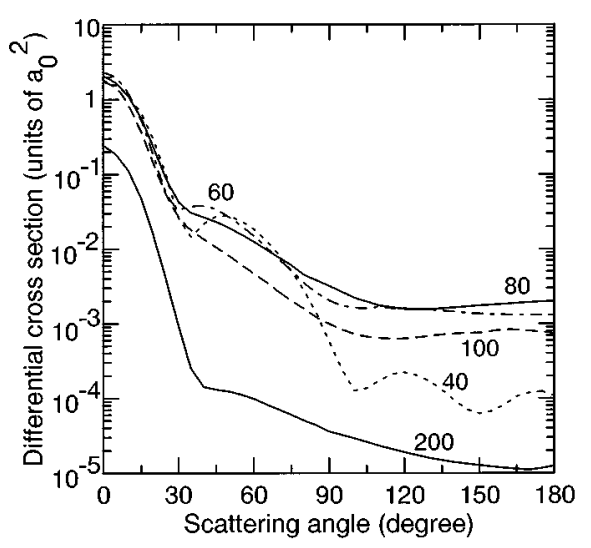

FIG. 4. Differential cross sections (in units of $a_{0}^{2}$ ) for rearrangement transition to the $\operatorname{Ps}(1 s)$ state in positron-helium scattering employing the present eight-state CCA scheme. The curves are labeled by different incident positron energies.

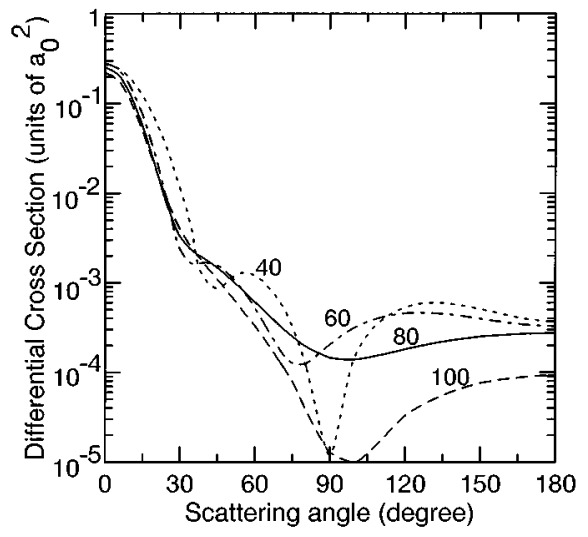

FIG. 5. Differential cross sections (in units of $a_{0}^{2}$ ) for rearrangement transition to the $\operatorname{Ps}(2 s)$ state in positron-helium scattering employing the present eight-state CCA scheme. The curves are labeled by different incident positron energies.

different from the eight-state results. This shows the necessity of the positronium atom states for obtaining convergence of the elastic and inelastic positron-helium scattering cross sections.

Finally, we present the differential capture cross sections to the $\operatorname{Ps}(1 s), \operatorname{Ps}(2 s)$, and $\operatorname{Ps}(2 p)$ states in positron-helium scattering using the present eight-state CCA scheme in Figs. 4,5 , and 6 , respectively.

At higher energies the differential cross sections for elastic and inelastic positron-helium scattering are expected to be similar to that of the electron-helium system, where the effect of exchange is expected to be small. By comparing our results with those of Refs. [10,17] we find that this general trend holds in the present calculation.

\section{B. Coincidence parameters}

We also studied the coincidence parameters $\lambda$ and $\chi$ as they are equally important for the complete determination of scattering. While differential cross section is the measure of the probability of scattering in different directions, the dimensionless coincidence parameters describe the state of the

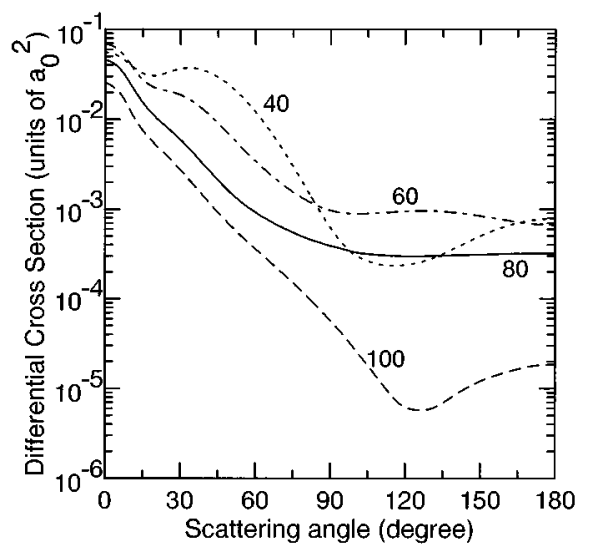

FIG. 6. Differential cross sections (in units of $a_{0}^{2}$ ) for rearrangement transition to the $\operatorname{Ps}(2 p)$ state in positron-helium scattering employing the present eight-state CCA scheme. The curves are labeled by different incident positron energies. 


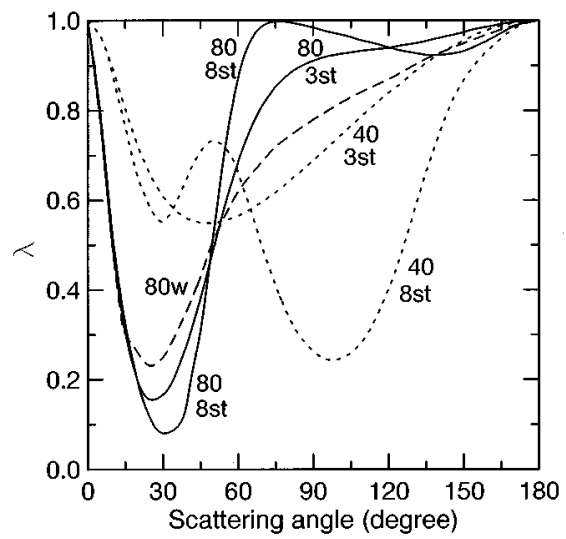

FIG. 7. Orientation parameter $(\lambda)$ for excitation to the $\mathrm{He}\left(1 s 2^{1} p\right)$ state in positron-helium scattering at incident positron energies 40 and $80 \mathrm{eV}$ employing the three- and eight-state CCA schemes of Fig. 2. The curves are labelled by different incident positron energies; 3st-present three-state CCA, 8st-present threestate CCA, 80w-three-state CCA from Ref. [10].

atoms after the collision. We calculated $\lambda$ and $\chi$ following Eminyan et al. [17] for transition to the $\operatorname{He}\left(1 s 2^{1} p\right)$ state. In Figs. 7 and 8 we plot the $\lambda$ and $\chi$ parameters, respectively, for transition to the $\operatorname{He}\left(1 s 2^{1} p\right)$ state employing the present eight-state CCA scheme as well as the three-state CCA scheme employing the following states: $\operatorname{He}(1 s 1 s)$, $\mathrm{He}\left(1 s 2^{1} s\right)$, and $\mathrm{He}\left(1 s 2^{1} p\right)$. For comparison we also present the results of the three-state CCA calculation of Ref. [10]. The present three-state result is in reasonable agreement with that of Ref. [10].

The nature of the alignment parameter $\chi$ according to our three-state CCA scheme at $80 \mathrm{eV}$ agrees well with that of Ref. [10] only up to $\theta=30^{\circ}$. We also got a broad maximum at $30^{\circ}$ as in Ref. [10], however, with a slightly higher value. But above $30^{\circ}$, our $\chi$ parameter varies continuously, giving a wavelike structure, while in Ref. [10] it is almost constant. With the inclusion of Ps-formation channels (eight-state CCA), the discrepancy between the present result and that of Ref. [10] increases, especially at larger angles.

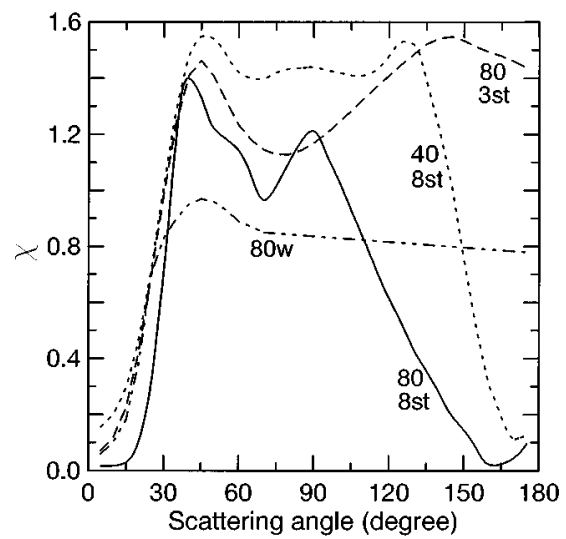

FIG. 8. Alignment parameter $(\chi)$ for excitation to the $\mathrm{He}\left(1 s 2^{1} p\right)$ state in positron-helium scattering at incident positron energies 40 and $80 \mathrm{eV}$ employing the three- and eight-state CCA schemes of Fig. 2. For notations, see Fig. 7.

\section{SUMMARY}

We have performed an eight-state CCA calculation for positron-helium scattering at medium energies $(40 \mathrm{eV}-200$ $\mathrm{eV}$ ) employing realistic wave functions for helium states [15] and exact Ps-atom wave functions. We present results for differential cross sections for (a) elastic scattering, (b) inelastic transition to $\mathrm{He}\left(1 s 2^{1} s\right)$ and $\mathrm{He}\left(1 s 2^{1} p\right)$ states of helium, and (c) rearrangement transition to $\operatorname{Ps}(1 s), \operatorname{Ps}(2 s)$, and $\operatorname{Ps}(2 p)$ states of the positronium atom. In addition, we present results for the coherent $\lambda$ and $\chi$ parameters for inelastic transition to the $\mathrm{He}\left(1 s 2^{1} p\right)$ state of helium. The results of the present study are consistent with the three-state calculation of Ref. [10].

\section{ACKNOWLEDGMENTS}

We thank Professor A. S. Ghosh for helpful discussions. The work is supported in part by the Conselho Nacional de Desenvolvimento-Científico e Tecnológico, Fundação de Amparo à Pesquisa do Estado de São Paulo, and Coordenação de Aperfeiçoamento de Pessoal do Nível Superior and Financiadora de Estudos e Projetos of Brazil, and the John Simon Guggenheim Memorial Foundation.
[1] T. C. Griffith, Adv. At. Mol. Phys. 22, 37 (1986).

[2] P. G. Coleman and J. T. Hutton, Phys. Rev. Lett. 45, 2017 (1980); L. S. Fornari, L. M. Diana, and P. G. Coleman, ibid. 51, 2276 (1983); D. Fromme, G. Kruse, W. Raith, and G. Sinapius, ibid. 57, 3031 (1986); T. S. Stein, W. E. Kauppila, V. Pol, J. H. Smart, and G. Jension, Phys. Rev. A 17, 1600 (1978); L. M. Diana, P. G. Coleman, D. L. Brooks, P. K. Pendleton, and D. M. Norman, ibid. 34, 2731 (1986); T. C. Griffith and G. R. Heyland, Phys. Rep. 39C, 169 (1978).

[3] K. F. Canter, P. G. Coleman, T. C. Griffith, and G. R. Heyland, J. Phys. B 6, L201 (1973); N. Overton, R. J. Mills, and P. G. Coleman, ibid. 26, 3951 (1993); M. Charlton and G. Laricchia, ibid. 23, 1045 (1990).

[4] D. R. Schulz, C. O. Reinhold, and R. E. Olson, Phys. Rev. A 40, 4947 (1989).
[5] E. F. Varracchio, J. Phys. B 23, L779 (1990).

[6] P. Khan, P. S. Mazumdar, and A. S. Ghosh, Phys. Rev. A 31, 1405 (1985); N. C. Deb, D. S. F. Crothers, and D. Fromme, J. Phys. B 23, L483 (1990).

[7] P. Mandal, A. S. Ghosh, and N. C. Sil, J. Phys. B 8, 2377 (1975).

[8] R. N. Hewitt, C. J. Noble, and B. H. Bransden, J. Phys. B 25, 557 (1992).

[9] S. L. Willis and M. R. C. McDowell, J. Phys. B 15, L31 (1982).

[10] S. L. Willis, J. Hata, M. R. C. Mcdowell, C. J. Joachain, and F. W. Byron, J. Phys. B 14, 2687 (1981).

[11] M. Kahali, S. K. Adhikari, D. Basu, and A. S. Ghosh, Chem. Phys. Lett. 239, 344 (1995) 
[12] S. K. Adhikari and A. S. Ghosh, Chem. Phys. Lett. 262, 460 (1996).

[13] P. Chaudhuri, S. K. Adhikari, and A. S. Ghosh, J. Phys. B 30, L81 (1997).

[14] H. R. J. Walters, J. Phys. B 21, 1893 (1988); S. K. Adhikari, A. S. Ghosh, and H. Ray, Chem. Phys. Lett. 222, 302 (1994); H. Ray, S. K. Adhikari, and A. S. Ghosh, ibid. 227, 429 (1994).

[15] T. G. Winter and C. C. Lin, Phys. Rev. A 12, 434 (1975).
[16] See, for example, S. K. Adhikari and K. L. Kowalski, Dynamical Collision Theory and Its Applications (Academic Press, Boston, 1991); S. K. Adhikari, Variational Principles and the Numerical Solution of Scattering Problems (John Wiley and Sons Inc., New York, in press).

[17] K. Bartschat, E. T. Hudson, M. P. Scott, P. G. Burke, and V. M. Burke, J. Phys. B 29, 2875 (1996); M. Eminyan, K. B. MacAdam, J. Slevin, and H. Kleinpoppen, ibid. 7, 1519 (1974). 